\title{
The mirtron miR-1010 functions in concert with its host gene SKIP to maintain synaptic homeostasis
}

\author{
Christopher Amourda ${ }^{1,4, *}$ and Timothy E. Saunders ${ }^{1,2,3, *}$ \\ ${ }^{1}$ Mechanobiology Institute, National University of Singapore, Singapore \\ ${ }^{2}$ Department of Biological Sciences, National University of Singapore, Singapore \\ ${ }^{3}$ Institute of Molecular and Cell Biology, A*Star, Proteos, Singapore \\ ${ }^{4}$ Lead Contact \\ *Correspondence: christopher.amourda@nus.edu.sg and dbsste@nus.edu.sg
}

\section{Summary}

Mirtrons are non-canonical miRNAs arising by splicing and debranching from short introns. A plethora of introns have been inferred by computational analyses as potential mirtrons. Yet, few have been experimentally validated and their functions, particularly in relation to their host genes, remain poorly understood. Here, we found that larvae lacking the mirtron miR-1010 are unable to grow properly and pupariate. We show that miR-1010 downregulates nAcR $\beta 2$. Increase of cortical nAcR $\beta 2$ mediated by neural activity elevates the level of intracellular $\mathrm{Ca}^{2+}$, which in turn activates CaMKII and, further downstream, the transcription factor Adf-1. We reveal that Adf-1 initiates the expression of SKIP, the host gene of miR-1010. Preventing synaptic potentials from overshooting their optimal range requires both SKIP to temper synaptic potentials (incoherent feedforward loop) and miR-1010 to reduce nAcR $\beta 2$ mRNA levels (negative feedback loop). Our results demonstrate how a mirtron, in coordination with its host gene, contributes to maintaining homeostasis.

Keywords: mirtron function, miR-1010, synaptic homeostasis, nicotinic receptor, Adf-1, incoherent feedforward loop, negative feedback loop 


\section{Introduction}

Since their discovery more than two decades ago (Lee, Feinbaum and Ambros, 1993; Wightman, Ha and Ruvkun, 1993), microRNAs (miRNAs) have been established as key cellular micromanagers. MiRNAs control nearly all cellular pathways, with a large subset being indispensable to development (Lucchetta, Carthew and Ismagilov, 2009; Carthew, Agbu and Giri, 2017; Mok et al., 2018). Canonical miRNAs are genetically encoded short (18-22 nucleotides) hairpin structures that are recognised, after transcription, by the RNAse type III endonuclease Drosha which cleaves the stem region in the nucleus (Denli, A.M., Tops, B.B.J., Plasterk, R.H.A., Ketting, R.F., Hannon, 2004; Gregory et al., 2004; Berezikov, 2011). MiRNAs are subsequently exported from the nucleus and undergo a final step of maturation before being functionally able to regulate the mRNA level of their target genes (Pasquinelli, Hunter and Bracht, 2005; Giri and Carthew, 2014; Carthew, Agbu and Giri, 2017; Chandra et al., 2017). Recent computational and experimental efforts identified a non-canonical miRNA maturation pathway in which introns are debranched from precursor mRNAs by the Lariat debranching enzyme and enter the miRNA processing pathway without requiring cleavage by Drosha (Okamura et al., 2007; Ruby, Jan and Bartel, 2007; Westholm and Lai, 2011). This new class of miRNAs were named mirtrons and numerous introns have, subsequently, been inferred as potential mirtrons (Chung et al., 2011; Ladewig et al., 2012; Wen et al., 2015). Further studies revealed that mirtrons are widespread across taxa and some show a high degree of conservation (Curtis, Sibley and Wood, 2012), implying important regulatory functions.

The repertoire of canonical miRNA genomic sources is characterised by its versatility. Indeed, miRNAs can be found as single or clustered transcriptional units bearing their own regulatory elements. MiRNAs are also found within introns of host genes both in sense or anti-sense orientations, indicating that their expression does not necessarily correlates with that of their host genes (Rodriguez et al., 2004; Martinez et al., 2008; Ozsolak et al., 2008; Isik and 
Berezikov, 2013). An important question arising from the genomic organisation of canonical miRNAs is: what is the reason for the emergence of mirtrons? It has been hypothesised that alternative miRNA processing pathways may be important in stressful conditions. Anaerobic conditions in tumors or exposure to hormones lead to a down-regulation or inhibition of the miRNA processing components (Merritt et al., 2008; Faggad et al., 2010). For example, Drosha mRNA level is reduced by around $50 \%$ in ovarian-cancer specimens. In such circumstances, pathways regulated by miRNAs are perturbed (Merritt et al., 2008). Although, the presence of mirtrons in such stressful conditions has not been documented, an attractive possibility is that mirtrons fulfil fundamental roles under stressful conditions to maintain cellular homeostasis (Curtis, Sibley and Wood, 2012). In particular, since mirtrons mature regardless of the level of Drosha, their processing and maturation should not be affected under stress as the transcription machinery remains functional. Alternatively, mirtrons may have emerged from mutation of short intronic sequences that evolved into hairpin structures (Berezikov et al., 2007; Curtis, Sibley and Wood, 2012). Importantly, the biological significance of intronic miRNAs and, especially, mirtrons must be understood with respect to the function of their host gene (Inui et al., 2018).

Here, we show that the mirtron miR-1010 regulates the level of the nicotinic acetylcholine receptor $\beta 2(\mathrm{nAcR} \beta 2)$ via a negative feedback loop whereby elevated nAcR $\beta 2$ results in increased miR-1010 levels. In the absence of miR-1010 larvae cease growth and do not pupariate. Elevated levels of $\mathrm{nAcR} \beta 2$ upon neural activity also triggers a homeostatic response whereby SKIP, the host gene of miR-1010, amplifies the Shal $\mathrm{K}^{+}$channel role in tempering membrane potentials (Diao, Waro and Tsunoda, 2009; Ping and Tsunoda, 2011). However, this negative feedforward response is dispensable, with viable adults emerging in SKIP mutants. Our work demonstrates that miR-1010, transcribed alongside SKIP, is involved in a critical 
negative feedback loop that decreases nAcR $\beta 2$ mRNA levels to restore homeostasis. Importantly, we deconstruct the underlying mechanisms of the feedforward and feedback loops that regulate the neuron potential, and we find that the presence of miR-1010 is vital in this process, in contrast to that of SKIP. Finally, we show that miR-1010 is upregulated upon exposure to nicotine. Therefore, we believe that our results will be contribute to further our understanding of nicotine-related disorders.

\section{Results and Discussion}

\section{MiR-1010, in contrast to its host gene SKIP, is indispensable to viability}

We brought our attention to the mirtron miR-1010 as homozygous mutants are lethal (Chen et al., 2014). MiR-1010 is located within the Shal potassium $\left(\mathrm{K}^{+}\right)$channel Interacting Protein (SKIP) gene, between exons 4 and 5 (Figures. 1A and S1). Replacing miR-1010 by a LoxP sequence does not affect embryogenesis as larvae hatch without any apparent defects and with similar viability as wild-type animals. However, mutant larvae are unable to grow; they retain a first instar larva size $(<0.5 \mathrm{mg})$ and fail to pupariate even after ten days of larval life (Figure. 1B). The ecdysone pathway, a steroid hormone controlling larval moulting (Baehrecke, 1996; Yamanaka, Rewitz and O'Connor, 2013), appears unaffected with the Ecdysone-induced protein 75B (E75B), the Prothoracicotropic hormone (PTTH), Phantom (Phm) and Disembodied (Dib) being expressed within wild-type ranges (Figures S2A-E). Furthermore, the insulin receptor (dInr) and the Drosophila insulin-like peptide 2 (Dilp2) are upregulated to counteract the lack of growth (Figures S2F and S2G). The standard level of chico (substrate of the insulin receptor (Boulan, Milán and Léoplold, 2016)), sNPF and ns3 (both involved in dilp2 secretion (Boulan, Milán and Léoplold, 2016)) concur with the insulin pathway and dilp2 secretion being operative in miR-1010 ${ }^{-/-}$(Figures S2H-J). Moreover, we observed no defects in food intake in miR-1010 ${ }^{-/-}$larvae (Figure 1C). Strikingly, the metabolic brake 4E-BP (Teleman, Chen and Cohen, 2005) shows a 10-fold increase directly at the onset of larval life and remains 
at a high level in miR-1010 mutant larvae as compared to wild-type larvae (Figure 1D). The high level of 4E-BP indicates that larvae sense a stressful condition and, as a result, growth is inhibited until returning to more adequate conditions (Teleman, Chen and Cohen, 2005). Prior to investigating the causes underlying the miR-1010-/- phenotype, we first sought to verify that the impeded growth is solely due to the lack of miR-1010 and not a result of an alteration of SKIP. For this purpose, we used a SKIP MiMIC $\left(\mathrm{SKIP}^{-/-}\right)$mutant where a triple stop codon is inserted within the first intron (Venken et al., 2011). Importantly, the MiMIC insertion does not prevent transcription but alters the translation of a given gene. Hence, the miR-1010 level remains at a wild-type level in $\mathrm{SKIP}^{-/-}$larvae (Figure S2K). Concordantly, $\mathrm{SKIP}^{-/-}$larvae show a mild timing phenotype and pupate with a $24 \mathrm{~h}$ delay (Figure 1B). These results indicate that the deficit in larval growth is due to a lack of miR-1010.
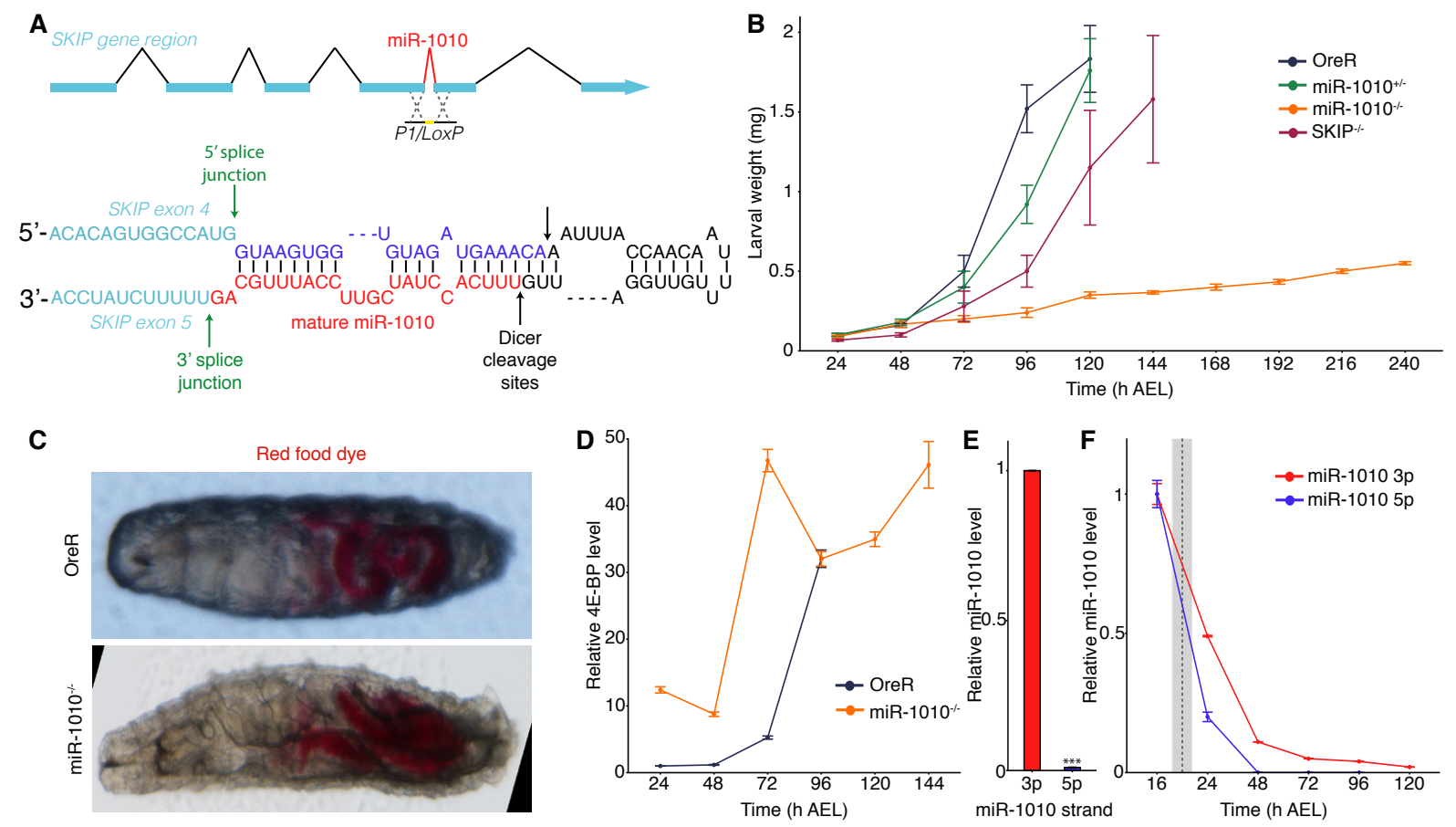

Figure 1. Larvae fail to grow and pupariate in miR-1010 ${ }^{-/-}$

(A) The mirtron miR-1010 is located in the SKIP gene, between the exons 4 and 5. The entire miR-1010 has been replaced by a LoxP sequence in the miR-1010 ${ }^{-/-}$line (top panel). MiR-1010 forms a hairpin loop and is debranched from SKIP by the splicing machinery and further matures as a normal miRNA (bottom panel). (B) Larval weight was measured directly after hatching until pupariation. OreR, miR-1010 ${ }^{+/-}$and $\mathrm{SKIP}^{-/-}$reached $\sim 2 \mathrm{mg}$ before pupariation. MiR-1010 $0^{-/-}$weight was recorded for 10 days but did not pupariate. (C) Stereoscope pictures of OreR and miR-1010 ${ }^{-/-}$larvae allowed to eat coloured yeast paste show no feeding behaviour 
defect. (D) Larval 4E-BP transcript levels measured by RT-qPCR in OreR and miR-1010-/. Fold changes are relative to OreR at 24h AEL. (E-F) MiR-1010 strand specific RT-qPCR shows a strong bias toward miR-1010 3p (E) and a high expression during embryogenesis before gradually decreasing during larval stages $(\mathbf{F})$. Expression level are relative to the expression at $16 \mathrm{~h} \mathrm{AEL}$. The dashed line indicates the average hatching time and the grey rectangle represents the standard deviation in hatching times at $25^{\circ} \mathrm{C}$. All values are means $\pm \mathrm{SD}(* * * \mathrm{P}<0.001, \mathrm{n}=$ at least 9 for each experiment).

\section{$\mathrm{nAcR} \beta 2$ is the main target of $\mathrm{miR}-1010$}

We next sought to identify the targets of miR-1010. In contrast to canonical miRNAs, mirtrons 3' arms are preferentially more stable than their 5' complement (Okamura et al., 2007). Consistent with this biased stability, we found only traces of miR-1010 5' strand throughout development (Figure 1E). Further, we observed that miR-1010 has its highest expression during mid-embryogenesis (16h AEL) and gradually decreases during larval development (Figure 1F). We next used a Gal4 driver inserted downstream of the SKIP regulatory region (SKIP $>$ Ga14) to locate the spatial expression of both SKIP and miR-1010. We assume here that SKIP and miR-1010 have the same expression pattern as miR-1010 arises from the debranching of SKIP introns. We found that SKIP and miR-1010 are expressed in the central nervous system (CNS) and more prominently in the axons emanating from the CNS (Figure 2A and Movie 1). Coupling this expression pattern with the computationally predicted targets (Lewis, Burge and Bartel, 2005; Kheradpour et al., 2007; Ruby et al., 2007; Ruby, Jan and Bartel, 2007) for the miR-1010 3' strand, we refined a list of targets and examined their expression in miR-1010 ${ }^{-/}$. Amongst 36 tested targets, we found that Drl-2, nAcR $\beta 2$ and CG3078 are (i) expressed at meaningful level (judged by the $\mathrm{Ct}$ values in qPCR experiments) and (ii) consistently overexpressed (generally $>2$-fold for all targets) in miR-1010 $0^{-/-}$throughout larval development (Figures 2B, 2D and S3a). Of these, only nAcR $\beta 2$ shows higher expression during embryogenesis (Figure S3B). Notably, the $\mathrm{nAcR} \beta 2$ is amongst the most prominently expressed nAcRs during early larval life and further the only subtype overexpressed in miR-1010-/ (Figures $\mathrm{S} 3 \mathrm{C}$ and $\mathrm{S} 3 \mathrm{D}$ ). We infer from these data that $\mathrm{nAcR} \beta 2$ plays a crucial role at this stage 
of development and accounts, in part, for the phenotype observed in miR-1010 $0^{-/}$. Alongside the predicted targets, we noticed that the mRNA levels of SKIP and Shal in miR-1010 ${ }^{-/}$larvae show a $\sim 3$-fold increase as compared to control larvae (Figure 2E). The increase in mRNA level translates into an increase in protein level for both $\mathrm{nAcR} \beta 2$ and SKIP (Figures S3E and S3F). Importantly, the nAcR $\beta 2$ protein level is slightly increased during embryogenesis but dramatically greater at larval stages in miR-1010 ${ }^{-/-}$(Figure S3E). SKIP has two shorter isoforms besides its full length protein (Diao, Waro and Tsunoda, 2009) and we observed an upregulation of both the full length and the SKIP3 isoform at larval stages (Figure S3F). SKIP3 has previously been shown to favour the slow inactivation mode of Shal (Diao, Waro and Tsunoda, 2009). These results suggest that miR-1010, its host gene, and targets are within the same regulatory pathway.

\section{Overexpression of $\mathrm{nAcR} \beta 2$ results in miR-1010 upregulation}

We next attempted to rescue the miR-1010 ${ }^{-/-}$phenotype by knocking down specific targets. Mutants for Drl-2 and CG3078 fail to rescue the miR-1010-- phenotype (Figure S4A). We were unable to perform similar rescue experiment for the $\mathrm{nAcR} \beta 2$ as the cytogenic locations of miR-1010 and nAcR $\beta 2$ are in close proximity (94A4 and 96A5, respectively) and therefore prevent chromosomal recombination. MiRNA deficiency essentially results in overexpression of its targets. We reasoned that overexpressing the coding sequence (i.e. without the 3'UTR bearing miR-1010 binding sites) of miR-1010 targets should phenocopy miR-1010 ${ }^{-/}$(Figure S4B). We did not impede larval growth as in miR-1010-/ (Figures S4C-S4E), nevertheless, panneural (Elav $>$ Gal4) overexpression of $n A c R \beta 2$ resulted in an interesting pattern whereby SKIP, miR-1010 and Shal are upregulated along with Drl-2 and CG3078 (Figure 2F). On the contrary, overexpression of nAcR $\beta 2$ in sensory neurons using Pickpocket Gal4 driver (Adams et al., 1998; Ainsley et al., 2008) (ppk>Gal4) did not increase the level of SKIP, miR-1010 and Shal 
(Figure 2F). These results indicate that miR-1010 is neuron specific. Overexpressing Drl-2 or CG3078 with Elav>Gal4 does not affect the levels of nAcR $\beta 2$, SKIP and Shal (Figures S4FS4G). Further, we noticed that the SKIP $>$ Gal4 driver line (when homozygous) exhibits a high level of $n A c R \beta 2$ accompanied by high levels of SKIP and Shal (Figure 2G). The SKIP $>$ Gal4 line is, however, homozygous viable. Therefore, this expression pattern indicates that SKIP transcription or introns debranching functions at a sub-optimal level in the SKIP $>$ Gal4 line. This phenotype can be partially rescued upon restoring a copy of a wild-type chromosome (Figure 2G). Last, we were able to rescue the larval growth defects in miR-1010 ${ }^{-/}$by expressing miR-1010 under the pan-neural driver Elav (Figures 2H and S4H-S4J). The mRNA levels of nAcR 32 , Shal, Drl-2 and CG3078 are brought down by the overexpression of miR-1010. However, the SKIP mRNA level is consistently higher $(\sim 10$-fold $)$ in the rescue line. This expression pattern is surprising and further experiments will be required to understand the reason underlying SKIP upregulation. Altogether, these experiments indicate that an increase in $\mathrm{nAcR} \beta 2$ gives rise to an upregulation of SKIP, miR-1010 and Shal genes, a necessity to maintain the neural circuit within acceptable conditions. The upregulation of Drl-2 and CG3078 upon $\mathrm{nAcR} \beta 2$ increase is intriguing. The most likely explanation is that the higher levels of Drl-2 and CG3078 in miR-1010 ${ }^{-/-}$larvae is simply a response to increased neural activity, not the direct result of a lack of miR-1010. CG3078 upregulation upon neural activity is plausible as its inferred function is to regulate $\mathrm{K}^{+}$channel activity (Gramates et al., 2017). However, the reason for Drl-2, an axon guiding protein (Grillenzoni et al., 2007; Sakurai et al., 2009; Reynaud et al., 2015), upregulation is more enigmatic and may be a response to axonal growth following increased activity. 

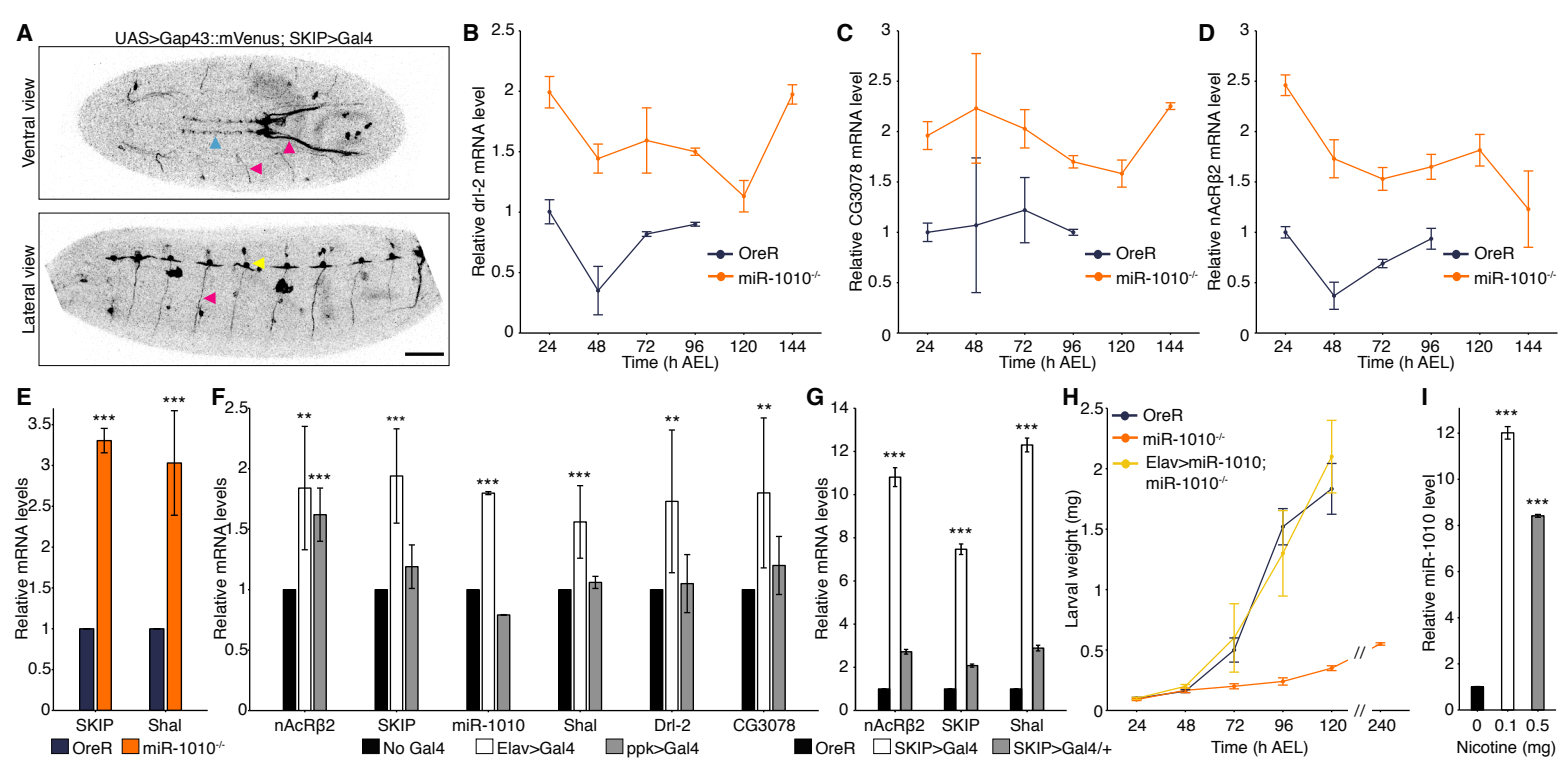

Figure 2. nAcR $\beta 2$, Drl-2 and CG3078 levels is elevated in miR-1010 ${ }^{-/-}$

(A) Confocal imaging of embryos expressing a membrane marker (UAS $>$ Gap43::mVenus) driven by SKIP $>$ Gal4. SKIP/miR-1010 are expressed in the CNS (blue arrowhead), in axons (pink arrowheads) emanating from the CNS and at neuromuscular junctions (yellow arrowhead). Scale bar is 50 $\mu \mathrm{m}$. (B-D) Drl-2 (B), CG3078 (C) and nAcR 32 (D) transcript levels (RT-qPCR) in miR-1010 ${ }^{-/ /}$(orange). Fold changes are relative to OreR (dark blue) at $24 \mathrm{~h}$ AEL. (E) SKIP and Shal transcript levels (RT-qPCR) in miR-1010 ${ }^{-/-}$relative to OreR larvae at $24 \mathrm{~h}$ AEL. (F) Transcripts levels (RT-qPCR) in nAcR $\beta 2$ overexpressed by Elav $>$ Gal4 (white) or ppk $>$ Gal4 (grey) relative to non-induce UAS $>$ nAcR $\beta 2$ (black) at 24h AEL. (G) nAcR 32 , SKIP and Shal transcript levels (RT-qPCR) in homozygous SKIP $>$ Gal4 (white) and in SKIP $>$ Gal4/+ (gray) relative to OreR (black) at $24 \mathrm{~h}$ AEL. (H) Larval weight was measured in OreR, miR$1010^{-/-}$and in Elav $>$Gal4/UAS $>$miR-1010; miR-1010 ${ }^{-/-}$rescue line. (i) miR-1010 levels upon exposure to $0.1 \mathrm{mg}$ (white) and $0.5 \mathrm{mg}$ (gray) of nicotine as compared to $0 \mathrm{mg}$ (black). All values are means $\pm \mathrm{SD}(* \mathrm{P}<0.05, * * \mathrm{P}<0.01, * * * \mathrm{P}<0.001, \mathrm{n}=$ at least 9 for each experiment $)$.

\section{Nicotine exposure elevates the level miR-1010}

nAcRs boost synaptic potentials and trigger the upregulation of cortical nAcRs (Ping and Tsunoda, 2011). These receptors are permeable to $\mathrm{Ca}^{2+}$ and the subsequent influx of $\mathrm{Ca}^{2+}$ activates the CaMKII. In turn, CaMKII triggers the expression of the Shal $\mathrm{K}^{+}$channel. Therefore, $\mathrm{K}^{+}$ions are released from neurons and temper membrane potentials. Shal and its interacting protein SKIP have been shown to be important members of a pathway required to stabilise these synaptic potentials (Diao, Waro and Tsunoda, 2009; Ping and Tsunoda, 2011). Despite the apparent importance of such a pathway, we found that neither knockout of Shal nor 
SKIP resulted in lethality (Figure S4K). Combining these results, we hypothesised that the mRNA level of $n A c R \beta 2$ is downregulated by miR-1010 and that this negative feedback works complementarily to SKIP and Shal to temper synaptic potentials. As previously described, SKIP is presumed to modulate Shal channels inactivation kinetics by favouring Shal slow inactivation mode (Tsunoda and Salkoff, 1995; Diao, Waro and Tsunoda, 2009; Ping et al., 2011). Crucially, however, our results suggest that the SKIP/Shal part of the pathway is only acting to temper the potential response and is not fundamental in restoring homeostasis. In contrast, the negative feedback loop mediated via miR-1010 is indispensable for returning the system to homeostasis after stimulation.

To further test this model, we exposed wild-type larvae to different doses $(0.1$ and $0.5 \mathrm{mg})$ of nicotine, agonist of nAcRs, for $24 \mathrm{~h}$. We saw a striking $\sim 10$-fold increase in miR-1010 levels for both dosage conditions (Figure 2I). This result shows that there exists a direct relationship between nAcRs activation and miR-1010 expression. Importantly, it suggests that miR-1010 plays a hitherto underappreciated role in regulating synaptic potentials under stress conditions.

\section{Adf-1 controls SKIP, miR-1010 and Shal expression}

We next examined the regulation of SKIP and Shal expression. Through computational analysis, we found that the Alcohol dehydrogenase transcription factor 1 (Adf-1) has predicted binding sequences in both Shal and SKIP regulatory regions (Messeguer et al., 2002; Farré et al., 2003) (Figure S5A). Further, Adf-1 is phosphorylated by CaMKII and is therefore a plausible candidate (Timmerman et al., 2013). It has also been demonstrated by ChIP-seq experiments that Adf-1 is phosphorylated upon neural activity and blocks Fas2 and (indirectly) Staufen to allow neuronal growth (Timmerman et al., 2013). We obtained the ChIP-seq results mentioned above and noticed that Adf-1 also binds to Shal and to a smaller extent to SKIP (Figure S5B). We confirmed these results by performing a ChIP-qPCR for Adf-1 and showed that Adf-1 
strongly binds Fas2, Shal and SKIP regulatory regions in OreR and miR-1010 ${ }^{+/-}$mutants. However, this binding is significantly reduced in miR-1010-/ (Figure 3A). Adf-1 has been shown to positively correlate with high Pol II-pausing indices (Timmerman et al., 2013). Therefore, we reasoned that in its non-phosphorylated state Adf-1 is bound to Shal and SKIP regulatory elements and prevents their transcription. Upon neural activity, Adf-1 is phosphorylated and releases Shal and SKIP expression. If our hypothesis is correct, we should then observe higher levels of SKIP, miR-1010 and Shal in Adf- $1^{-/-}$larvae. The majority of Adf- $1^{-/}(\sim 80 \%)$ fail to complete embryogenesis (Dezazzo et al., 2000). Those that survive to larval stages do not grow noticeably and display a phenotype reminiscent to that of miR-1010-/larvae (Figure 3B). We performed qPCR on Adf- $1^{-/-}$that reached the larval stage and we saw a general increase in mRNA levels for all candidates (Figure 3C). However, our results are quite variable, a phenomenon unsurprising given the pleiotropic function of Adf-1 (Dezazzo et al., 2000; Timmerman et al., 2013). Adf-1 promotes neuronal growth upon neural activity by shutting down Fas2 and Staufen. Mechanism(s) must be in place to prevent detrimental overgrowth. By having Adf-1 also regulate Shal and SKIP, the system couples growth to neural activity. This mechanism introduces an incoherent feedforward loop to ensure robust growth in response to neural activity (Figure 3D).

A

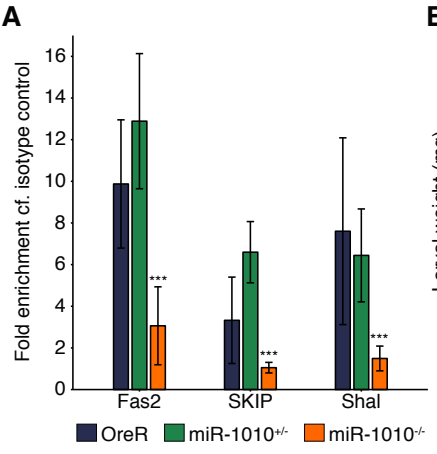

B

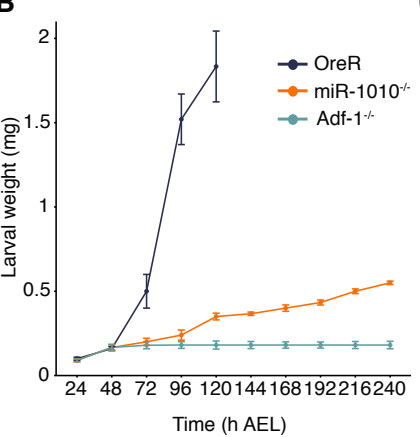

C

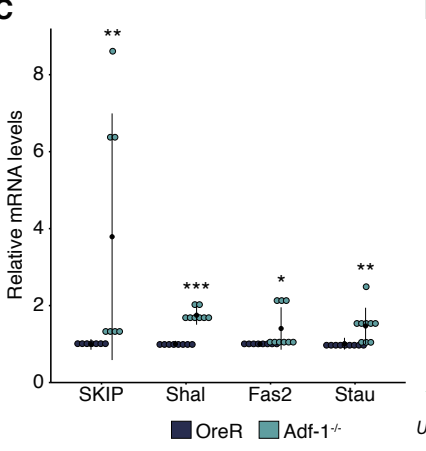

D

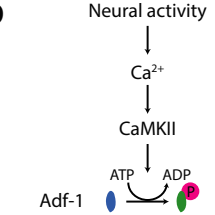
is 
measured in Adf- $1^{-/-}$(turquoise) relative to OreR (dark blue) at 24h AEL. Black dots and lines represent, respectively, the mean and the standard deviation for each condition. (D) Model of Adf-1-mediated coupling of neural activity and growth. Adf- 1 is represented in blue in its nonphosphorylated form and in green upon phosphorylation. All values are means $\pm \mathrm{SD}\left({ }^{*} \mathrm{P}<0.05\right.$, $* * \mathrm{P}<0.01, * * * \mathrm{P}<0.001, \mathrm{n}=$ at least 9 for each experiments).

\section{MiR-1010, in concert, with SKIP is necessary to maintain synaptic homeostasis}

We have revealed here that $\mathrm{nAcR} \beta 2$, Shal, SKIP and miR-1010 form a network that contains an incoherent feedforward loop coupled with a negative feedback loop (Figure 4A). Shal and SKIP are necessary to counteract the influx of positively charged ions upon neural activity. Nevertheless, Shal and SKIP are not sufficient to maintain membrane potentials within an optimal range and the regulation of $\mathrm{nAcR} \beta 2$ by miR-1010 appears to be necessary to preserve synaptic homeostasis. Therefore, the mirtron miR-1010 and its host gene SKIP appear to work in tandem to ensure synaptic homeostasis (Figure 4B). To test this conclusion, we used massaction kinetics to model the average long-term voltage potential response due to the two interaction loops outlined in Figure 4A after activation of nAcR $\beta 2$ (see Supplementary Note for details). We see that loss of SKIP or partial loss of miR-1010 results in delayed return of the potential to homeostatic levels (Figure 4C), consistent with our observed delay in developmental time (Figure 1B). However, complete loss of miR-1010 results in the potential staying active over a long period (Figure 4C), consistent with the observed stress response seen in $\mathrm{miR}-1010^{-/-}$. The modelling reveals that miR-1010 is effectively acting like a switch, controlling whether nAcR $\beta 2$ has high or low expression, and this switch-like behaviour is dependent on miR-1010 expression levels (see Supplementary Note for further discussion). The model predicts that the average membrane potential response will also be higher in miR-1010 and $\mathrm{SKIP}^{-/-}$and it will be interesting to test this, for example using a GCaMP reporter.

We have studied the role of miR-1010 in a developmental context but it is important to note that exposure to nicotine results in a dramatic increase of miR-1010 level in wild-type larvae. Hence, this pathway is likely not restricted to development and applies to later stages of 
life. Deciphering the relevance of miR-1010 regulation in nicotine-related disorder (such as nicotine addiction or Alzheimer disease) would constitute exciting avenues of research. Our work sheds light on the coherence between the functions of mirtrons and their host genes. In this case, SKIP and miR-1010 act in a coordinated fashion to efficiently maintain homeostasis. We predict that (i) such regulatory loops are not restricted to synaptic homeostasis and (ii) homologous pathways exist in higher organisms. MiR-1010 shares sequence homologies with the mammalian miR-412 (Ibáñez-Ventoso, Vora and Driscoll, 2008). However, miR-412 is not a mirtron and does not seem to directly regulate nAcRs (from computationally predicted targets). Rather, we believe that identifying a functional homolog would help to further understand the pertinence of homeostasis control by mirtrons.
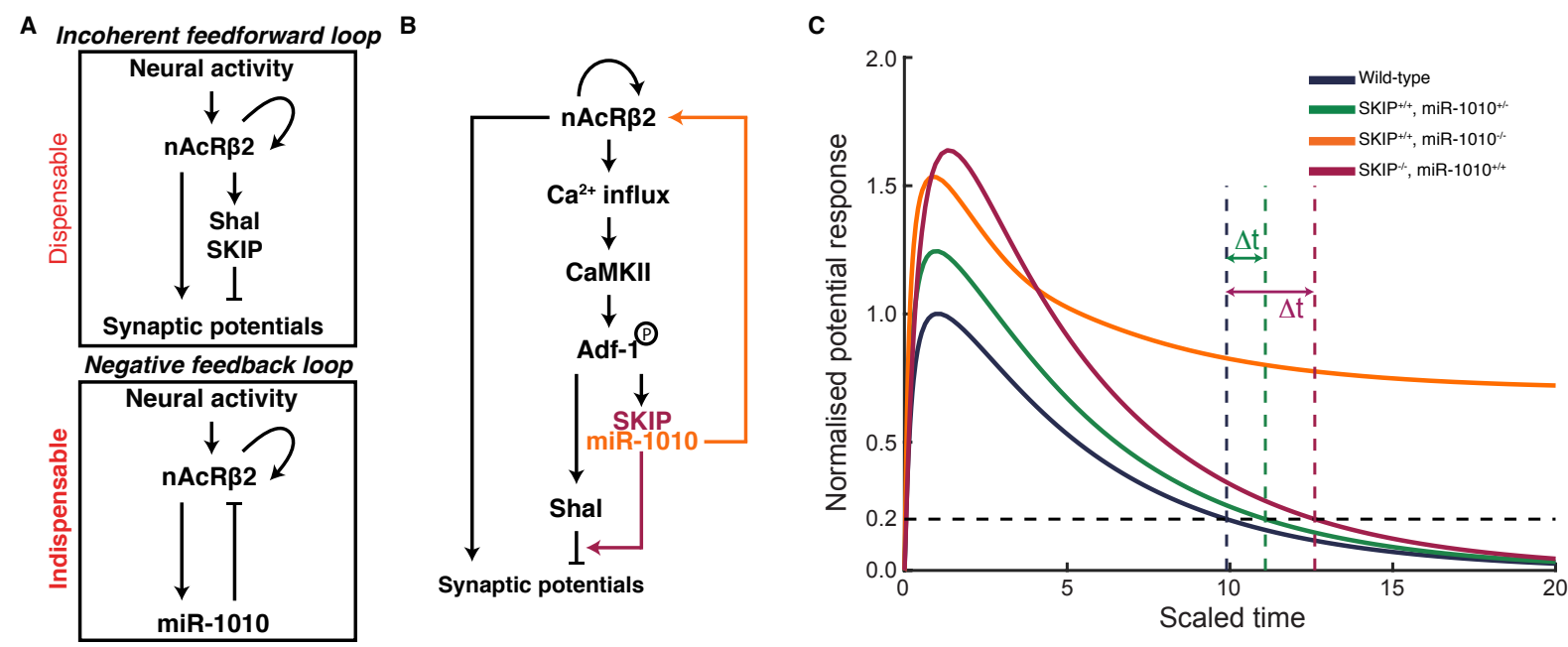

\section{Figure 4. miR-1010 controls synaptic homeostasis by downregulating nAcR $\beta 2$}

(A) nAcR $\beta 2$ is part of incoherent feedforward loop to temper synaptic potentials and a negative feedback loop through which miR-1010 downregulates nAcR $\beta 2$ upon receptor activation. (B) Both loops work cooperatively to prevent synaptic potentials from overshooting their optimal range. (C) Long-term average potential response obtained by mass-action kinetic simulation of model outlined in (A). Resting potential defined to 0 (corresponding to $\sim-70 \mathrm{meV}$ ). Curves normalised to maximum potential in wild-type simulation (parameters and description in Supplementary Note). Time is scaled relative to the lifetime of $\mathrm{nAcR} \beta 2 . \Delta \mathrm{t}$ represents delay in potential in miR- $1010^{+/-}$and $\mathrm{SKIP}^{-/-}$to return to less than $20 \%$ of peak response in wild-type conditions. 


\section{Acknowledgments:}

We thank Victor Corces and Stephen Cohen for sharing precious reagents and data. We acknowledge Katsutomo Okamura and all Saunders' lab members for fruitful discussions. This work was supported by the National Research Foundation Singapore under an NRF Fellowship to T.E.S. (NRF2012NRF-NRFF001-094).

\section{Author Contributions:}

C.A. designed the study, performed the experiments, analysed the data and wrote the manuscript. T.E.S. performed the mathematical modelling, aided in the study design and statistical analysis, and contributed to writing the manuscript. Both authors agree to the final version of the manuscript.

The authors declare no competing financial interest. 


\section{References}

Adams, C. M. et al. (1998) 'Ripped Pocket and Pickpocket, Novel Drosophila DEG/ENaC Subunits Expressed in Early Development and in Mechanosensory Neurons', The Journal of cell biology, 140(1), pp. 143-152.

Ainsley, J. A. et al. (2008) 'Sensory mechanisms controlling the timing of larval developmental and behavioral transitions require the Drosophila $\mathrm{DEG} / \mathrm{ENaC}$ subunit, Pickpocket1', Developmental Biology, 322(1), pp. 46-55. doi: 10.1016/j.ydbio.2008.07.003.

Baehrecke, E. H. (1996) 'Ecdysone signaling cascade and regulation of Drosophila metamorphosis.', Archives of insect biochemistry and physiology, 33, pp. 231-244. doi: 10.1002/(SICI)1520-6327(1996)33:3/4<231::AID-ARCH5>3.0.CO;2-V.

Berezikov, E. et al. (2007) 'Mammalian Mirtron Genes', Molecular Cell, 28(2), pp. 328-336. doi: 10.1016/j.molcel.2007.09.028.

Berezikov, E. (2011) 'Evolution of microRNA diversity and regulation in animals.', Nature reviews. Genetics. Nature Publishing Group, 12(12), pp. 846-60. doi: 10.1038/nrg3079.

Boulan, L., Milán, M. and Léoplold, P. (2016) 'The Systemic Control of Growth', Cold Spring Harbor perspectives in biology, 7, p. a019117.

Carthew, R. W., Agbu, P. and Giri, R. (2017) 'MicroRNA function in Drosophila melanogaster', Seminars in Cell and Developmental Biology. Elsevier Ltd, 65, pp. 29-37. doi: 10.1016/j.semcdb.2016.03.015.

Chandra, S. et al. (2017) 'Role of miRNAs in development and disease: Lessons learnt from small organisms', Life Sciences. Elsevier Inc., 185(5), pp. 8-14. doi: 10.1016/j.lfs.2017.07.017.

Chen, Y. et al. (2014) 'Resource Systematic Study of Drosophila MicroRNA Functions Using a Collection of Targeted Knockout Mutations', Developmental Cell. Elsevier Inc., 31(6), pp. 784-800. doi: 10.1016/j.devcel.2014.11.029.

Chung, W. et al. (2011) 'Computational and experimental identification of mirtrons in Drosophila melanogaster and Caenorhabditis elegans', Genome Research, 21, pp. 286-300. doi: 10.1101/gr.113050.110.

Curtis, H. J., Sibley, C. R. and Wood, M. J. A. (2012) 'Mirtrons, an emerging class of atypical miRNA', Wiley Interdisciplinary Reviews: RNA, 3(5), pp. 617-632. doi: 10.1002/wrna.1122.

Denli, A.M., Tops, B.B.J., Plasterk, R.H.A., Ketting, R.F., Hannon, G. J. (2004) 'Processing of pri- mary microRNAs by the microprocessor complex', Nature, 432(7014), pp. 231-235. Available at: http://eutils.ncbi.nlm.nih.gov/entrez/eutils/elink.fcgi?dbfrom=pubmed\&id=15531879\&retmod $\mathrm{e}=$ ref\&cmd=prlinks\%0Apapers3://publication/doi/10.1038/nature03049.

Dezazzo, J. et al. (2000) 'nalyot, a Mutation of the Drosophila Myb-Related Adf1 Transcription Factor, Disrupts Synapse Formation and Olfactory Memory', Neuron, 27, pp. $145-158$.

Diao, F., Waro, G. and Tsunoda, S. (2009) 'Fast inactivation of Shal (Kv4) K+ channels is 
regulated by the novel interactor SKIP3 in Drosophila neurons', Molecular and Cellular Neuroscience. Elsevier Inc., 42(1), pp. 33-44. doi: 10.1016/j.mcn.2009.05.003.

Faggad, A. et al. (2010) 'Prognostic significance of Dicer expression in ovarian cancer-link to globalmicroRNA changes and oestrogen receptor expression', The Journal of pathology, 220, pp. 382-391. doi: 10.1002/path.

Farré, D. et al. (2003) 'Identification of patterns in biological sequences at the ALGGEN server: PROMO and MALGEN', Nucleic Acids Research, 31(13), pp. 3651-3653. doi: $10.1093 /$ nar/gkg605.

Giri, R. and Carthew, R. W. (2014) 'microRNAs suppress cellular phenotypic heterogeneity', Cell Cycle, 13(10), pp. 1517-1518. doi: 10.4161/cc.29013.

Gramates, L. S. et al. (2017) 'FlyBase at 25: Looking to the future', Nucleic Acids Research, 45(D1), pp. D663-D671. doi: 10.1093/nar/gkw1016.

Gregory, R. I. et al. (2004) 'The Microprocessor complex mediates the genesis of microRNAs', Nature, 432(7014), pp. 235-240. doi: 10.1038/nature03120.

Grillenzoni, N. et al. (2007) 'Respective roles of the DRL receptor and its ligand WNT5 in Drosophila mushroom body development', Development, 134(17), pp. 3089-3097. doi: 10.1242/dev.02876.

Ibáñez-Ventoso, C., Vora, M. and Driscoll, M. (2008) 'Sequence relationships among C. elegans, D. melanogaster and human microRNAs highlight the extensive conservation of microRNAs in biology', PLoS ONE, 3(7). doi: 10.1371/journal.pone.0002818.

Inui, M. et al. (2018) 'Dissecting the roles of miR-140 and its host gene', Nature Cell Biology, 20(5), pp. 516-518. doi: 10.1038/s41556-018-0077-4.

Isik, M. and Berezikov, E. (2013) 'Expression Pattern Analysis of MicroRNAs in Caenorhabditis elegans’, pp. 129-141. doi: 10.1007/978-1-62703-083-0_11.

Kheradpour, P. et al. (2007) 'Reliable prediction of regulator targets using 12 Drosophila genomes', Genome Research, 17(12), pp. 1919-1931. doi: 10.1101/gr.7090407.

Ladewig, E. et al. (2012) 'Discovery of hundreds of mirtrons in mouse and human small RNA data’, Genome Research, 22(9), pp. 1634-1645. doi: 10.1101/gr.133553.111.

Lee, R. C., Feinbaum, R. L. and Ambros, V. (1993) 'The C . elegans Heterochronic Gene lin4 Encodes Small RNAs with Antisense Complementarity to \& II-14', Cell, 75, pp. 843-854. doi: 10.1016/0092-8674(93)90529-Y.

Lewis, B. P., Burge, C. B. and Bartel, D. P. (2005) 'Conserved seed pairing, often flanked by adenosines, indicates that thousands of human genes are microRNA targets', Cell, 120(1), pp. 15-20. doi: 10.1016/j.cell.2004.12.035.

Lucchetta, E. M., Carthew, R. W. and Ismagilov, R. F. (2009) 'The Endo-siRNA pathway is essential for robust development of the Drosophila embryo', PLoS ONE, 4(10). doi: 10.1371/journal.pone.0007576.

Martinez, N. J. et al. (2008) 'Genome-scale spatiotemporal analysis of Caenorhabditis elegans 
microRNA promoter activity Genome-scale spatiotemporal analysis of Caenorhabditis elegans microRNA promoter activity', Genome Research, pp. 2005-2015. doi: 10.1101/gr.083055.108.

Merritt, W. M. et al. (2008) 'Dicer, Drosha, and Outcomes in Patients with Ovarian Cancer', The New England journal of medicine, 359(25), pp. 2641-2650. doi: 10.1056/NEJMoa0803785.Dicer.

Messeguer, X. et al. (2002) 'PROMO: Detection of known transcription regulatory elements using species-tailored searches', Bioinformatics, 18(2), pp. 333-334. doi: 10.1093/bioinformatics/18.2.333.

Mok, G. F. et al. (2018) 'miR-133-mediated regulation of the Hedgehog pathway orchestrates embryo myogenesis', 2, pp. 1-10. doi: 10.1242/dev.159657.

Okamura, K. et al. (2007) 'The Mirtron Pathway Generates microRNA-Class Regulatory RNAs in Drosophila’, Cell, 130(1), pp. 89-100. doi: 10.1016/j.cell.2007.06.028.

Ozsolak, F. et al. (2008) 'Chromatin structure analyses identify miRNA promoters', Genes and Development, 23, pp. 3172-3183. doi: 10.1101/gad.1706508.sion.

Pasquinelli, A. E., Hunter, S. and Bracht, J. (2005) 'MicroRNAs: A developing story', Current Opinion in Genetics and Development, 15(2), pp. 200-205. doi: 10.1016/j.gde.2005.01.002.

Ping, Y. et al. (2011) 'Shal/Kv4 channels are required for maintaining excitability during repetitive firing and normal locomotion in Drosophila', PLOS ONE, 6(1), pp. 15-20. doi: 10.1371/journal.pone.0016043.

Ping, Y. and Tsunoda, S. (2011) 'Inactivity-induced increase in nAChRs upregulates Shal K+ channels to stabilize synaptic potentials', Nature Neuroscience, 15(1), pp. 90-97. doi: 10.1038/nn.2969.

Reynaud, E. et al. (2015) 'Guidance of Drosophila Mushroom Body Axons Depends upon DRL-Wnt Receptor Cleavage in the Brain Dorsomedial Lineage Precursors', Cell Reports. The Authors, 11(8), pp. 1293-1304. doi: 10.1016/j.celrep.2015.04.035.

Rodriguez, A. et al. (2004) 'Identification of mammalian microRNA host genes and transcription units', Genome Research, 14(10 A), pp. 1902-1910. doi: 10.1101/gr.2722704.

Ruby, J. G. et al. (2007) 'Evolution, biogenesis, expression, and target predictions of a substantially expanded set of Drosophila microRNAs', Genome Research, 17(12), pp. 18501864. doi: 10.1101/gr.6597907.

Ruby, J. G., Jan, C. H. and Bartel, D. P. (2007) 'Intronic microRNA precursors that bypass Drosha processing', Nature, 448(7149), pp. 83-86. doi: 10.1038/nature05983.

Sakurai, M. et al. (2009) 'Differentially Expressed Drl and Drl-2 Play Opposing Roles in Wnt5 Signaling during Drosophila Olfactory System Development', Journal of Neuroscience, 29(15), pp. 4972-4980. doi: 10.1523/JNEUROSCI.2821-08.2009.

Teleman, A. A., Chen, Y. W. and Cohen, S. M. (2005) '4E-BP functions as a metabolic brake used under stress conditions but not during normal growth', Genes and Development, 19(16), pp. 1844-1848. doi: 10.1101/gad.341505. 
Timmerman, C. et al. (2013) 'The Drosophila Transcription Factor Adf-1 (nalyot) Regulates Dendrite Growth by Controlling FasII and Staufen Expression Downstream of CaMKII and Neural Activity', Journal of Neuroscience, 33(29), pp. 11916-11931. doi: 10.1523/JNEUROSCI.1760-13.2013.

Tsunoda, S. and Salkoff, L. (1995) 'Genetic analysis of Drosophila neurons: Shal, Shaw, and Shab encode most embryonic potassium currents', The Journal of Neuroscience, 15(3), pp. 1741-1754. Available at: http://www.ncbi.nlm.nih.gov/pubmed/7891132.

Venken, K. J. T. et al. (2011) 'MiMIC: a highly versatile transposon insertion resource for engineering Drosophila melanogaster genes', Nature Methods, 8(9), pp. 737-743. doi: 10.1038/nmeth.1662.

Wen, J. et al. (2015) 'Analysis of Nearly One Thousand Mammalian Mirtrons Reveals Novel Features of Dicer Substrates', PLoS Computational Biology, 11(9), p. e1004441. doi: 10.1371/journal.pcbi.1004441.

Westholm, J. O. and Lai, E. C. (2011) 'Mirtrons: MicroRNA biogenesis via splicing', Biochimie. Elsevier Masson SAS, 93(11), pp. 1897-1904. doi: 10.1016/j.biochi.2011.06.017.

Wightman, B., Ha, I. and Ruvkun, G. (1993) 'Posttranscriptional regulation of the heterochronic gene lin-14 by lin-4 mediates temporal pattern formation in C. elegans', Cell, 75(5), pp. 855-862. doi: 10.1016/0092-8674(93)90530-4.

Yamanaka, N., Rewitz, K. F. and O'Connor, M. B. (2013) 'Ecdysone Control of Developmental Transitions: Lessons from Drosophila Research', Annual Review of Entomology, 58(1), pp. 497-516. doi: 10.1146/annurev-ento-120811-153608. 